\title{
TTR
}

Traduction, terminologie, rédaction

\section{La recherche en enseignement de la traduction au Canada : survol historique et enjeux actuels}

\section{Álvaro Echeverri}

Volume 30, numéro 1-2, 1er semestre-2e semestre 2017

La traductologie en mouvement

Translations Studies: A Forward-Moving Discipline

URI : https://id.erudit.org/iderudit/1060022ar

DOI : https://doi.org/10.7202/1060022ar

Aller au sommaire du numéro

Éditeur(s)

Association canadienne de traductologie

ISSN

0835-8443 (imprimé)

1708-2188 (numérique)

Découvrir la revue

Citer cet article

Echeverri, Á. (2017). La recherche en enseignement de la traduction au Canada : survol historique et enjeux actuels. TTR, 30(1-2), 149-171.

https://doi.org/10.7202/1060022ar

\section{Résumé de l'article}

Les projets éducatifs répondent aux besoins d'un groupe social en particulier. $\mathrm{Au}$ Canada, la formation des traducteurs a été conçue dans le but, entre autres, de contribuer aux efforts d'amélioration de la qualité de la langue française (Juhel, 1982; Horguelin, 1984). Les stratégies didactiques utilisées par les premiers formateurs ont ainsi favorisé l'instauration d'une tradition d'enseignement à visée corrective qui résiste à l'épreuve du temps et qui a essaimé au-delà des frontières canadiennes. Des universitaires canadiens sont en effet à l'origine de deux des principales approches d'enseignement de la traduction, toutes traditions confondues : l'approche comparative (Vinay et Darbelnet, 1958) et l'approche par objectifs d'apprentissage (Delisle, 1980). Après avoir retracé l'émergence de ces approches et décrit ce qui a contribué à leur succès, je m'interrogerai sur les enjeux actuels relatifs à la recherche sur l'enseignement de la traduction. 


\title{
La recherche en enseignement de la traduction au Canada : survol historique et enjeux actuels
}

\author{
Álvaro Echeverri \\ Université de Montréal
}

\section{Résumé}

Les projets éducatifs répondent aux besoins d'un groupe social en particulier. Au Canada, la formation des traducteurs a été conçue dans le but, entre autres, de contribuer aux efforts d'amélioration de la qualité de la langue française (Juhel, 1982; Horguelin, 1984). Les stratégies didactiques utilisées par les premiers formateurs ont ainsi favorisé l'instauration d'une tradition d'enseignement à visée corrective qui résiste à l'épreuve du temps et qui a essaimé au-delà des frontières canadiennes. Des universitaires canadiens sont en effet à l'origine de deux des principales approches d'enseignement de la traduction, toutes traditions confondues: l'approche comparative (Vinay et Darbelnet, 1958) et l'approche par objectifs d'apprentissage (Delisle, 1980). Après avoir retracé l'émergence de ces approches et décrit ce qui a contribué à leur succès, je m’interrogerai sur les enjeux actuels relatifs à la recherche sur l'enseignement de la traduction.

Mots-clés : enseignement de la traduction, behaviorisme, objectifs d'apprentissage, tradition, Canada

\begin{abstract}
Educational efforts satisfy the needs of specific social groups. In Canada, translator training was conceived as a means, among others, to improve the quality of the French language (Juhel, 1982; Horguelin, 1984). The teaching strategies launched by the first trainers gave rise to a training tradition based on corrective measures that has survived the passage of time and expanded beyond the Canadian frontiers. Canadian scholars have proposed two of the main translator training approaches all traditions considered: the comparative approach (Vinay and Dalbernet, 1958) and the learning by objectives approach (Delisle, 1980). After presenting the context in which these approaches emerged and described what contributed to their success, I will discuss the current issues that arise with regard to research on translation teaching.
\end{abstract}

Keywords: translator training, behaviorism, learning by objectives, tradition, Canada 


\section{Introduction}

Anthony Pym et quelques-uns de ses collègues de l'Universitat Rovira i Virgilli, en Espagne, plus précisément en Catalogne, ont publié en 2003 le rapport d'un symposium en ligne sur l'enseignement de la traduction (cf. Pym et al., 2003). Les organisateurs du symposium avaient rédigé une série des questions qu'ils ont posées à cinq traductologues qui s'intéressent à l'enseignement de la traduction. ${ }^{1}$ Une des questions visait à savoir qui devrait enseigner la traduction. À celle-ci, Daniel Gouadec a répondu :

As to who should teach, the answer seems to be: whoever is willing to do so. Training translators (as distinct from teaching translation) and, what is more (or worse), training technical translators does not carry the kind of glamour associated with teaching more 'intellectual' courses and, basically, training teachers of languages. Anyone who writes a thesis on such subjects may not expect to get excessively fast promotion. No wonder there is such a dearth of trained teachers. (2003, p. 13)

Cette réponse est doublement intéressante parce qu'elle renvoie à la fois à la problématique de la formation des traducteurs à la traduction et à celle de la recherche sur l'enseignement de la traduction. En ce qui concerne la formation des traducteurs, il s'agit d'une tâche partagée entre des traducteurs de métier et des professeurs de carrière. De manière générale, ces deux groupes ne suivent pas une formation exclusivement conçue pour enseigner la traduction. Par rapport à la recherche sur l'enseignement de la traduction, du moins au Canada, il semble que Gouadec ait raison: depuis une vingtaine d'années, des thèses de doctorat portant sur le sujet ont été soutenues au pays, mais leurs auteurs ne consacrent pas leurs principaux efforts de recherche au thème de l'enseignement de la traduction. Le type de recherche dont je parle ici doit être entendu comme l'acquisition ou le développement de nouvelles connaissances ou de nouvelles pratiques; pour qu'il y ait recherche, il faut qu'il y ait eu un problème et une question sans réponse a priori, et que les solutions proposées soient des hypothèses de solution (Van der Maren, 2003, p. 37). Cette définition permet d'opérer la distinction entre la recherche d'une part et l'intervention d'expert d'autre part. L'intervention d'expert, très présente dans la tradition canadienne de formation des

1. Il s'agissait des personnes suivantes: Roberto Mayoral, Daniel Gouadec, Christiane Nord, Brian Mossop et Don Kiraly. 
traducteurs, ne vise ni l'acquisition ni le développement de nouvelles connaissances. L'expert utilise plutôt ses connaissances pour proposer les solutions les plus pertinentes à des problèmes déjà rencontrés ou étudiés (ibid.). Comme nous le verrons dans les pages qui suivent, la tradition canadienne de formation des traducteurs s'est établie sur le plan international grâce aux nombreux travaux proposés par des experts dont le but est l'amélioration de la qualité des traductions en français.

Après un bref rappel de la fonction sociale de la traduction au Canada, je retracerai la façon dont la traduction a fait son entrée dans les universités canadiennes et l'émergence de la tradition canadienne en formation des traducteurs. Je discuterai ensuite de l'état de la recherche sur la formation des traducteurs au Canada en prenant comme toile de fond l'application que Jean Delisle a faite de l'approche d'apprentissage par objectifs à l'enseignement de la traduction.

\section{Le rôle social de la traduction au Canada}

Comme tout projet éducatif, la formation des traducteurs au Canada répond à des besoins sociaux très précis que Denis Juhel a bien exprimé dans Bilinguisme et traduction au Canada : rôle sociolinguistique de la traduction (1982). Selon Juhel, la traduction assume trois principales fonctions au Canada : une fonction utilitaire, une fonction politique et une fonction dissimilatrice. La fonction utilitaire serait très présente dans la traduction de textes que Juhel appelle "courants» (1982, p. 60), comme des modes d'emploi d'appareils et d'autres textes produits par des entreprises privées ou des organisations qui ne relèvent pas d'instances gouvernementales. La fonction politique viserait à compenser un déficit de rédacteurs francophones au sein de la fonction publique. Quant à la fonction dissimilatrice, elle permettrait au gouvernement du Québec de se servir de la traduction afin de promouvoir l'usage de la langue française au sein de la population et d'assurer la qualité du français (ibid.).

Les premiers cours de traduction offerts au Canada ont été créés par des professionnels chevronnés souhaitant transmettre leur savoirfaire à des traducteurs en quête de perfectionnement. Préoccupés par la qualité linguistique des traductions publiées dans les journaux et dans la documentation publicitaire, des traducteurs d'expérience membres de l'Association des traducteurs du Québec ont commencé à offrir des cours de perfectionnement à leurs propres collègues vers 
1940. Le résultat a donné lieu à une tradition d'enseignement de la traduction, au Québec et au Canada, se démarquant par un intérêt à «induire de faits concrets de langage les règles ou les lois générales régissant la pratique de la traduction" (Delisle, 1990, p. 65).

L'établissement de cette tradition s'est organisé autour de deux axes, comme l'indique Paul Horguelin (1984, p. 26) : la formation des traducteurs et l'organisation de la profession. Pierre Daviault fait figure de proue dans cette tradition, et ses travaux, présentés sous forme des notes de traducteur, offrent un exemple de la visée corrective caractéristique des débuts de la formation des traducteurs au pays. Son livre Langage et traduction (1963) constitue un répertoire d'expressions et de mots anglais dont la traduction en français peut s'avérer difficile et pour lesquels des traductions commentées sont proposées. Les répertoires tels que celui de Daviault, que j’assimile aux contributions d'experts plutôt qu'à de la recherche scientifique, sont très présents dans la formation des traducteurs canadiens. Des ouvrages comme En français dans le texte de Robert Dubuc (1994 et 2000) de même que Le traducteur averti (2005) et Le traducteur encore plus averti (2016) de François Lavallée, notamment, en sont de bons exemples.

Après avoir fait son entrée à l'université au début des années 1970, cette tradition a pris un tournant comparatif auquel elle reste encore associée. En 2005, Christine Durieux déclarait qu'en matière d'enseignement de la traduction, le Canada «reste attaché à la mise en regard de ces deux langues, comme par un effet de miroir, l'une se reflétant dans l'autre» (p. 42). Dans son article, Durieux présente des arguments en faveur de l'innovation dans la formation des traducteurs. Cependant, à mon avis, les approches corrective et comparative adoptées au Canada étaient des stratégies adéquates pour le contexte social qui les a vues apparaître. Ces stratégies sont en place depuis presque quatre-vingts ans et elles ont été conçues afin d'améliorer la qualité linguistique des traductions à un moment particulier de l'histoire. Elles ont certainement apporté des résultats concrets, car la traduction et les traducteurs ne sont plus perçus comme la grande menace qui guettait le français entre la fin du XVIII e siècle et le début du $\mathrm{XX}^{\mathrm{e}}$ siècle. Déterminer l'adéquation de ces stratégies à la formation des traducteurs d'aujourd'hui et de demain constitue un des défis à relever pour tous ceux qui s'intéressent au sujet, qu'ils soient membres de l'industrie de la traduction ou universitaires. 


\section{L'entrée de la traduction à l'université}

Il s'avère pertinent de revenir sur les premiers pas de la traduction, en tant quactivité professionnelle, dans le contexte universitaire canadien. Au Canada, le marché de la traduction a comme caractéristique principale la prédominance de la traduction de l'anglais vers le français ${ }^{2}$, particulièrement dans la communication administrative et commerciale. En conséquence, la qualité du français écrit, dans ces domaines, reste fortement influencée par la qualité linguistique des traductions. Au Québec en particulier, dans le débat sur la qualité de la langue, la traduction et les traducteurs ont été au centre des discussions à partir de la fin du XVIII ${ }^{e}$ siècle. Paul Horguelin, notamment, fait référence au préjudice que le journal bilingue la Gazette du Québec, fondé en 1764, a porté à la qualité du français :

la langue du journalisme et du commerce est contaminée par les traducteurs improvisés de ce journal [...] «langue de traduction» caractérisée par des structures reproduisant celles de l'anglais, des tournures et expressions calquées, des anglicismes qui vont s'enraciner dans l'usage. (1984, p. 24)

La démarche corrective entamée au $\mathrm{XIX}^{\mathrm{e}}$ siècle avec la publication d'ouvrages comme Le manuel des difficultés les plus communes de la langue française de l'abbé Thomas Maguire (1841) ou le Recueil des expressions vicieuses et des anglicismes les plus fréquents de Jules Fabien Gingras (1860) s'est poursuivie au XX $\mathrm{XX}^{\mathrm{e}}$ siècle dans des travaux comme L'expression juste en traduction (1931) de Pierre Daviault. Selon Horguelin, cette démarche corrective s'est matérialisée dans toutes les initiatives qui ont mené à la création de l'Office de la langue française en 1961.

Paradoxalement, la traduction figure également au premier plan parmi les stratégies proposées pour redresser la situation. Comme l'exprimait Sherry Simon (1989, p. 62) pour parler de ce paradoxe, «la traduction agit négativement sur la langue; la traduction agit positivement dans un contexte de redressement linguistique». La traduction incarne à la fois une des sources des problèmes de

2. Comme l'explique Geneviève Mareschal, «[1]a prédominance du français sur le marché de la traduction [au Canada] a [...] influencé la conception des cursus, où la traduction de l'anglais vers le français (composante version) constitue l'élément central des apprentissages. Le passage actif vers l'anglais (composante thème) noccupe pas une place importante dans la formation. Suivant les programmes, il peut faire l'objet de quelques cours, parfois facultatifs, ou en être totalement absent» (2005, p. 252). 
la qualité du français et représente l'un des fers de lance des efforts de francisation, d'amélioration de la qualité du français et de défense du statut du français en tant que langue officielle. Vers 1940, la formation des traducteurs fait partie de la stratégie mise en place par la Société des traducteurs du Québec pour assurer la professionnalisation des traducteurs. Comme on peut le déduire en lisant Les Alchimistes des langues: la Société des traducteurs du Québec (1940-1990) de Jean Delisle (1990), la traduction a fait son entrée dans les universités canadiennes après que des traductrices et des traducteurs formés sur le tas aient souhaité perfectionner leur art et voir leurs compétences reconnues. Dans les premières cohortes d'étudiants et d'étudiantes, les secrétaires bilingues étaient bien représentées. Les formateurs de la première heure ont conçu leur tâche en termes de quête du bilinguisme parfait, et les cours de base ou de perfectionnement offerts aux traducteurs visaient la réduction des fautes de français repérées dans les textes traduits.

C'est à Montréal, grâce à la Société de traducteurs de Montréal, que les premières initiatives de formation des traducteurs ont vu le jour, en 1940. Visant à la fois la reconnaissance sociale de la profession et l'amélioration de la qualité des traductions, la Société de traducteurs de Montréal offrait des cours de perfectionnement à ses membres. Comme Delisle (1990, p. 6-7) l'a bien documenté, les artisans de l'organisation de ces cours ont été Georges Panneton et Jeanne Grégoire. Selon Delisle, Georges Panneton est à l'origine d'une tradition canadienne en formation de traducteurs dont les principaux représentants sont Pierre Daviault, Jean-Paul Vinay et Jean Darbelnet; le nom de Jean Delisle lui-même doit être ajouté à cette liste. La formation des traducteurs à l'époque se concevait principalement sous le prisme des études sur le bilinguisme et se donnait comme objectif la transformation des «faux bilingues» en de «vrais bilingues». Les vrais bilingues doivent se distinguer par leur capacité à dissocier les deux langues de travail et par leur capacité à déceler les principales difficultés à surmonter afin de produire des traductions de qualité. D'après Delisle,

[q]uant aux faux bilingues [...], leur aptitude à tenir la plume du traducteur n'a jamais été à la hauteur de leur bonne volonté. Ignorant les règles qui régissent le passage d'une langue à une autre, et incapables de les déduire par eux-mêmes, ils sont à l'origine des bourdes tristement désopilantes qui jettent le discrédit sur la profession tout entière. (ibid., p. 7) 
Cette transformation devait s'opérer par l'établissement d'autant de différences que possible entre les deux langues officielles dans le but d'exploiter la richesse expressive du français et d'éviter de tomber dans le réflexe morphologique, ou mimétisme, qui consiste, pour le traducteur, «à choisir systématiquement entre plusieurs équivalents lexicaux tous acceptables celui dont la forme ressemble le plus au mot original» (Delisle et Fiola, 2013, p. 669). Cette manière de procéder mine la richesse du français étant donné que ses ressources expressives ne sont pas convenablement exploitées. Une telle visée explique bien pourquoi un nombre considérable d'ouvrages didactiques issus de cette tradition prennent la forme de traités de stylistique comparée, de notes de traducteurs, de recueils de «difficultés de traduction» ou de «fautes de traduction» colligées au fil du temps par des traducteurs ou par des formateurs d'expérience.

Les premiers programmes de formation complète en traduction ont été créés à la fin des années 1960 à l'Université de Montréal, à l'Université Laval et à l'Université d'Ottawa (Mareschal, 2005, p. 250). Comme le signale Geneviève Mareschal, ces programmes et ceux qui ont suivi «sont tous plus ou moins coulés dans le même moule» (2005, p. 252), dans la mesure où ils ont été conçus «pour répondre à des besoins de bilinguisation officielle» (ibid.) et qu'ils visent «essentiellement le marché national»(ibid.). Ainsi, les diplômés des universités canadiennes reçoivent «une formation très comparable » (ibid.). Grâce au volet de perfectionnement linguistique offert dans les programmes de formation, les futurs traducteurs sont sensibilisés au rôle sociolinguistique qu'ils seront appelés à jouer.

L'entrée de la traduction à l'université coïncide notamment avec les projets sociaux, politiques et linguistiques de modernisation du Québec ayant mené à l'adoption des lois linguistiques qui ont marqué l'horizon politique québécois de l'époque ${ }^{3}$. Dans un tel contexte, la traduction devait contribuer à l'amélioration de la qualité du français, et les approches corrective et comparative se sont révélées efficaces pour remplir cette fonction à ce moment précis. D'abord, il fallait répertorier les difficultés de traduction pour en rendre les traducteurs conscients. C'est ce que Pierre Daviault a accompli dans son ouvrage Langage et traduction (1963). C'est aussi grâce à Daviault que l'université a ouvert ses portes à la traduction en 1936. C'est en

3. Rappelons que la Loi pour promouvoir la langue française au Québec (« loi 63 ») a été adoptée en 1969, la Loi sur la langue officielle (« loi 22 »), en 1974, et la Charte de la langue française (« loi $101 »)$, en 1977. 
effet à l'Université d'Ottawa que le premier cours de traduction de niveau universitaire a été donné, par Daviault lui-même. Il s'agissait d'un cours du soir offert principalement aux candidats aux postes de la fonction publique. Ce cours menait à l'obtention d'un certificat et il a été offert pendant vingt-six ans. Avec son cours, Daviault a contribué à la consolidation de la tradition qui a valu au Canada une place privilégiée dans le panorama mondial de la formation des traducteurs.

\section{La tradition canadienne en enseignement de la traduction}

Comme l'affirme André Lefevere (1977, p. 1), les traditions ne sont pas le produit du hasard. Elles sont créées par des personnes qui partagent des objectifs similaires pendant des années, des décennies, voire des siècles. Lefevere distingue quatre catégories de personnes appartenant à chacune des époques d'une tradition : les précurseurs, les pionniers, les maîtres et les disciples. Les précurseurs sont reconnus comme tels a posteriori par les membres de la tradition. Au Canada, la tradition de formation des traducteurs trouve ses origines dans les efforts des traducteurs professionnels. Elle se poursuit avec la collaboration entre professionnels et universitaires issus de disciplines comme la linguistique et l'enseignement de langues, et se consolide et vit un moment clé de son histoire vers la fin des années 1960 grâce à la création des premiers programmes universitaires. La création de ces programmes, en plus de marquer l'entrée définitive de la formation des traducteurs dans le cursus universitaire canadien, consolide l'étude de la traduction en tant que domaine de recherche.

Ainsi, si l'on reprend les catégories de Lefevere (1977) et qu'on les applique à la tradition de l'enseignement de la traduction au Canada, les précurseurs seraient Pierre Daviault, Georges Panneton et Jeanne Grégoire. Ces deux derniers sont à l'origine des premiers cours du soir que la Société des traducteurs de Montréal offrait à ses membres. Ces cours ont été repris et intégrés quelques années plus tard par l'Université McGill. Panneton et Grégoire sont également les fondateurs, vers 1940, de l'Institut de traduction de Montréal, qui sera par la suite annexé à l'Université de Montréal. D'après Delisle (1990, p. 66), Georges Panneton a en outre le mérite d'être le premier à avoir proposé en 1945 certains des procédés de traduction qui feront la gloire de La stylistique comparée de Vinay et Darbelnet, quelque dix ans plus tard. 
Après les précurseurs viennent les pionniers, qui ont la responsabilité d'écrire le manifeste de la tradition. Le rôle de pionniers dans la tradition canadienne de l'enseignement de la traduction revient à Jean-Paul Vinay et Jean Darbelnet, coauteurs de la Stylistique comparée de l'anglais et du français: méthode de traduction (1958). La stylistique comparée a fourni le cadre théorique sur lequel se sont structurés et consolidés les premiers programmes universitaires de formation des traducteurs au Canada et elle est encore présente dans certains programmes. La réputation internationale de la tradition canadienne en matière d'enseignement de la traduction repose en grande partie sur le travail de ces pionniers. L'histoire nous apprend en effet que Vinay et Darbelnet ont été très actifs dans la création des premiers programmes de traduction à l'Université Laval, à l'Université McGill, à l'Université d'Ottawa et à l'Université de Montréal (Kumbe, 2017, p. 63).

Les pionniers ouvrent le chemin aux maîtres. Les maîtres reprennent une partie du programme des pionniers, et leur travail représente le modèle par lequel les disciples, les derniers membres de la tradition, vont mesurer leur succès. Dans la tradition canadienne, le statut de maître revient à Jean Delisle, dont le manuel L'analyse du discours comme méthode de traduction : initiation à la traduction française des textes pragmatiques anglais. Théorie et pratique, paru en 1980, constitue l'une des plus importantes contributions à l'enseignement de la traduction, toutes traditions confondues ${ }^{4}$. Dans sa typologie des approches d'enseignement de la traduction, Dorothy Kelly (2005) met en première place l'approche par objectifs proposée par Delisle en 1980. Cette approche est celle qui a permis, pour la première fois, la présentation systématique des contenus d'un cours d'initiation à la traduction. De plus, Delisle a innové en concevant des activités pédagogiques qui n'impliquent pas la traduction d'un texte. Avec son manuel, Delisle a relevé un pari exceptionnel. Il a conçu une méthode d'initiation à la traduction des textes pragmatiques réunissant: 1) l'expérience des traducteurs professionnels sous la forme des difficultés de traduction; 2) les analyses linguistiques propres aux comparatistes; 3) les principes de la théorie interprétative, adaptés

4. L'influence de Jean Delisle à l'enseignement de la traduction ailleurs qu'au Canada s'observe dans divers pays, entre autres en Espagne. Amparo Hurtado Albir, notamment, souligne que Delisle a jeté les bases d'une formation des traducteurs organisée autour d'objectifs d'apprentissage, tout en permettant aux étudiants d'intérioriser le processus de traduction (2015, p. 276). 
à la traduction, suivant les préceptes de l'École de Paris; 4) les fondements de l'approche par objectifs qui a marqué le monde de l'éducation dans la deuxième moitié du $\mathrm{XX}^{\mathrm{e}}$ siècle.

Je me concentrerai dans ce qui suit sur les fondements théoriques en lien avec les sciences de l'éducation, qui sont à la base du succès de L'analyse du discours comme méthode de traduction. Lorsqu'il a proposé son manuel, en 1980, Delisle a mis en évidence les limites de la stylistique comparée qui s'imposait à l'époque et a ainsi provoqué un changement radical dans la formation des traducteurs au Canada. En fait, Delisle lui-même nous invite à revisiter les propositions pédagogiques qui s'éternisent :

Quand on retrace l'histoire de l'enseignement de la traduction au pays, il faut marquer d'une pierre blanche l'année 1958, date de parution de la Stylistique comparée du français et de l'anglais. Cet ouvrage fondateur aura cinquante ans l'an prochain. Tous s'accordent à reconnaitre qu'il a encore son utilité, mais qu'il a pris quelques rides. Plus les années passent, plus il devient urgent de le réviser et de l'actualiser. JeanPaul Vinay et Jean Darbelnet (1904-1994) ont su établir un pont entre la théorie et la pratique sans se détacher des faits concrets de langage. (2007, p. 85)

Revisitant lui-même son manuel, Jean Delisle en propose une nouvelle mouture en 1993 sous le titre La traduction raisonnée. Deux autres éditions suivront, en 2003 et en 20135. Chaque nouvelle édition offre un nombre d'objectifs d'apprentissage supérieur à celui des précédentes. Toutefois, les fondements théoriques de l'ouvrage restent les mêmes. De nos jours, il serait sans doute difficile de trouver un manuel didactique si pérenne, tous domaines confondus.

Je propose deux explications à la pérennité de la proposition didactique et pédagogique de Jean Delisle. La première tient à sa qualité et à son adéquation au contexte canadien. J'y reviendrai au paragraphe suivant. La deuxième est que les initiatives de recherche sur l'enseignement de la traduction au Canada n'arrivent pas à in-

5. Mentionnons que l'édition de 2003 a été réalisée avec la participation d'Alain René, traducteur au gouvernement du Canada et chargé de cours à l'Université d'Ottawa. Quant à l'édition de 2013, elle a été réalisée en collaboration avec Marco A. Fiola, professeur à 1'Université Ryerson, et elle regroupe en outre des contributions de Georges L. Bastin (Université de Montréal), Georges Farid (Université du Québec en Outaouais), Aline Francœur (Université Laval), Noëlle Guilloton (Office québécois de la langue française), André Guyon (Bureau de la traduction du gouvernement du Canada), Charles Le Blanc (Université d'Ottawa) et Elizabeth Marshman (Université d'Ottawa). 
fluer de manière déterminante sur les habitudes des formateurs, qui ont recours au manuel par conviction ou par tradition. Quoi qu'il en soit, du point de vue du progrès des connaissances en sciences de l'éducation, le milieu ne semble pas avoir de problème avec une approche pédagogique, l'apprentissage par objectifs, qui est très bien adaptée à la situation de l'enseignement de la traduction au Canada de 1980.

En ce qui concerne la qualité et l'adéquation du manuel au contexte canadien, force est de reconnaître que l'approche didactique proposée dans La traduction raisonnée mérite le succès qu'elle connaît. En cherchant dans les sciences de l'éducation un cadre théorique, Delisle a trouvé dans l'approche par objectifs des arguments épistémologiques qui validaient sa proposition didactique aux yeux de la communauté traductologique des années 1980. Un détour interdisciplinaire similaire à celui fait par Delisle permet de voir qu'il a réussi un pari très risqué. Il a pu marier la nature positiviste du behaviorisme dans lequel baigne l'approche par objectifs et le mentalisme phénoménologique de la théorie du sens. ${ }^{6}$

L'adéquation au rôle social que la traduction doit jouer au Canada est un autre facteur qui explique le succès et la pérennité de La traduction raisonnée. Comme je l'ai évoqué, la traduction joue un rôle important dans les efforts de maintien de la qualité du français au Canada. Ainsi, la plupart des programmes universitaires de formation de traducteurs incluent des cours de perfectionnement linguistique et de rédaction française. Ces cours portent des intitulés divers: Difficultés du français écrit, Interférences linguistiques, Rédaction professionnelle, etc. Conçue pour l'initiation à la traduction professionnelle, La traduction raisonnée consacre une bonne partie de son contenu aux difficultés de traduction. Dans l'édition de 2013, un peu plus de la moitié de l'ouvrage (en nombre de pages) est consacré au répertoire de difficultés de traduction. En ce sens, le manuel s'inscrit dans la même lignée que les travaux de Daviault réunis dans Langage et traduction (1963). Delisle fait d'ailleurs allusion à cette

6. L'apprentissage par objectifs représente le véhicule didactique sur lequel s'est déployée la toute première grande théorie de l'apprentissage. Cependant, les connaissances en sciences de l'éducation ont évolué; pendant que le nombre de difficultés (les objectifs spécifiques dans la terminologie de Delisle) augmente, le support théorique emprunté aux sciences de l'éducation, l'aspect qui distingue La traduction raisonnée d'autres manuels, "a pris quelques rides", pour reprendre l'expression utilisée par Delisle (2007, p. 86) en parlant de La stylistique comparée de Vinay et Darbelnet. 
particularité de la formation des traducteurs au Canada dans un extrait de La traduction raisonnée (2013) où il commente le contenu du manuel Le traducteur averti de François Lavallée (2005) et cite son auteur :

Le traducteur averti (2005) se révélera aussi d'une grande utilité. Son auteur, François Lavallée, l'a rédigé dans le même esprit que celui dans lequel nous avons élaboré les objectifs spécifiques de la Traduction raisonnée, en général, et l'OS 29 sur le mot juste, en particulier : comme "une chasse aux tours idiomatiques», mais «non pas comme on fait la chasse aux bisons, mais comme on fait la chasse aux papillons : pour enrichir sa collection» (Lavallée, 2005 : xxi). Ce recueil [Le traducteur averti] s'inscrit dans la lignée de ceux qu'a publiés en son temps le maître traducteur et pionnier de l'enseignement de la traduction au Canada, Pierre Daviault. Il se situe à mi-chemin entre le guide de traduction et le dictionnaire de difficultés. (Delisle et Fiola, 2013, p. 297-298)

En somme, il peut y avoir autant de répertoires de difficultés qu'il y a de traducteurs, et autant de répertoires d'erreurs commises par les étudiants qu'il y a de formateurs. Delisle ne souhaite pas reprendre toutes les difficultés répertoriées par Daviault, pas plus que Lavallée (2005) ne veut copier celles colligées par Dubuc (1994 et 2000), et ainsi de suite. Il faut l'avouer, la liste des ouvrages correctifs est particulièrement riche en contexte québécois. Ces ouvrages, dont la section «Difficultés d'ordre lexical» de la Traduction raisonnée fait partie, enrichissent les «boîtes à outils» des formateurs et offrent des possibilités d'autoformation tant aux futurs traducteurs qu'aux traducteurs en exercice.

La grande force de La traduction raisonnée se trouve manifestement dans la pertinence de ses objectifs généraux, qui synthétisent de manière magistrale les connaissances que les étudiants de niveau débutant doivent acquérir pour traduire des textes pragmatiques. À mon sens, les 28 premiers objectifs de l'édition 2013 constituent en eux-mêmes le manuel d'initiation à la traduction; les 47 objectifs suivants constituent quant à eux un excellent guide de traduction et un répertoire très complet de difficultés de traduction pour la formation des traducteurs au Canada.

Comme je l'ai mentionné plus haut, la pérennité de la proposition didactique de Jean Delisle pourrait aussi s'expliquer par l'absence d'un regard critique comme celui que Delisle lui-même a posé sur la Stylistique comparée de Vinay et Darbelnet. L'approche de formation par objectifs d'apprentissage demeure ainsi la base 
théorique du manuel d'enseignement de la traduction le plus utilisé au pays. Delisle a été le premier à proposer une approche de l'enseignement de la traduction fondée à la fois sur une théorie reconnue de l'apprentissage, le behaviorisme, et sur une approche de l'enseignement de la traduction, l'approche interprétative (Delisle, 1992, p. 38). Au behaviorisme, il a emprunté le concept d'objectif d'apprentissage en lui conférant un rôle central dans son cadre théorique. Les travaux de Benjamin Bloom (1975) et de Robert Mager (1977), deux auteurs reconnus pour l'adaptation qu'ils ont proposée de concepts propres à la psychologie behavioriste aux domaines de l'apprentissage et de l'enseignement, sont d'ailleurs cités dans l'introduction de la dernière édition de La traduction raisonnée (Delisle et Fiola, 2013, p. 20). Le concept d'objectif d'apprentissage tel qu'il est entendu dans La traduction raisonnée rappelle également un tenant central du behaviorisme: l'idée qu'une théorie du comportement ne peut se donner comme objectif que l'étude de comportements observables (ce qui est en nette opposition avec les théories mentalistes, qui cherchent à expliquer ce qui se produit dans la tête des personnes). Ainsi, selon Delisle et Fiola,

l'objectif spécifique (OS) est un énoncé formulé en termes de comportements observables qui décrit le plus précisément possible les résultats auxquels doivent conduire une ou plusieurs activités pédagogiques à l'intérieur d'un programme d'études ou d'un cours. (2013, p. 20; je souligne)

Dans cette perspective, l'attention du formateur est principalement dirigée vers la performance des apprenants. Les processus et les stratégies qui permettent à l'apprenant d'atteindre une telle performance passent au deuxième plan (Tardif, 1997, p. 63).

Les théories de l'apprentissage, tout comme les sociétés, ont évolué depuis les années 1980. La société industrielle et manufacturière dans laquelle est né le concept d'apprentissage par objectifs au milieu du $\mathrm{XX}^{\mathrm{e}}$ siècle a fait place à une société qu'on appelle la société du savoir centrée sur l'information et les services (Doughty, 2006; Jones et Idol, 1990). L'apprentissage par objectifs propose la division des contenus en petites unités qu'on présente aux apprenants en espérant qu'ils puissent reproduire le tout en situation de pratique ou d'évaluation. Toute l'idée des comportements observables découle de ce principe. Un comportement observable correspond à la reproduction correcte que les étudiants fournissent des contenus présentés au préalable en petites doses par les enseignants. La 
possibilité de décrire clairement les apprentissages visés reste le grand avantage de l'apprentissage par objectifs. Tant les formateurs que les apprenants peuvent ainsi se faire une idée claire des attentes réciproques. Le travail de conception et d'opérationnalisation des objectifs de même que les critères d'évaluation sont favorisés par la conception d'objectifs d'apprentissage généraux et spécifiques. Cette contribution de l'approche proposée par Delisle à l'enseignement de la traduction reste d'actualité, bien que les limites de l'approche par objectifs soient connues (Gimeno Sacristán, 1982; Louis, Jutras et Hensler, 1996). Elles sont principalement liées au découpage des unités de contenu et au fait qu'elles soient étudiées de façon détachée les unes des autres. Les formateurs se voient contraints de couvrir un nombre déterminé d'objectifs dans une période donnée, et les étudiants manquent parfois de temps pour donner un sens à ce qu'ils doivent apprendre. En conséquence, l'apprentissage se réduit souvent à des exercices de mémorisation.

Dans certains pays, l'enseignement de la traduction a évolué parallèlement aux sciences de l'éducation, en tenant compte de théories telles que le cognitivisme, le constructivisme et le socioconstructivisme qui, après le behaviorisme, sont venues enrichir les connaissances sur l'apprentissage et l'enseignement (Gauthier et Tardif, 2012). Des traductologues ont adapté leurs approches d'enseignement de la traduction en fonction de ces théories, grâce à des initiatives de recherche concertées et continues qui se prolongent dans le temps et qui se caractérisent par des projets consacrés exclusivement à la question de la formation de traducteurs. Ces projets sont menés par des chercheurs individuels ou des groupes de recherche. En guise d'exemple, on peut mentionner les propositions didactiques conçues en Espagne par Amparo Hurtado Albir. Les manuels de Hurtado Albir, structurés autour d'objectifs généraux et d'objectifs spécifiques, tiennent compte également des outils d'évaluation et des paramètres de l'évaluation. De plus, les objectifs spécifiques ne sont pas nécessairement liés à des difficultés de traduction précises. Les méthodes didactiques de Hurtado Albir sont passées d'une planification de contenu combinant les objectifs d'apprentissage et l'approche par tâches (1999) à une approche par compétences dans Aprender a traducir del francés al español: competencias y tareas para la iniciación a la traducción [Apprendre à traduire du français vers l'espagnol: compétences et tâches pour l'initiation à la traduction] (2015). Dans cet ouvrage, Hurtado Albir 
vante la nature constructiviste de son approche, qui suit les principes pédagogiques de l'approche par compétences $(2015$, p. 9). Un autre exemple de programme de recherche systématique consacré à l'enseignement de la traduction qui mérite dêtre mentionné est celui de Donald Kiraly, en Allemagne. Depuis la publication de son ouvrage Pathways to Translation (1995), Kiraly a en effet consacré l'ensemble de ses publications et de ses travaux de recherche à la formation des traducteurs.

\section{La recherche sur l'enseignement de la traduction au Canada : un domaine "orphelin"}

En 2005, dans un numéro de la revue Meta consacré à l'enseignement de la traduction, Geneviève Mareschal présentait de manière détaillée l'histoire et l'organisation des programmes de formation des traducteurs au Canada. Une section de son article est consacrée à la recherche. Fait intéressant, l'auteure ne parle pas de recherche sur l'enseignement de la traduction, mais de recherche sur la traduction ou la traductologie en général. Ses propos confirment cependant que la pédagogie de la traduction fait partie des sujets de recherche traductologiques :

Il suffit de jeter un coup d'œil aux titres des thèses de maîtrise et de doctorat pour voir combien les travaux et les intérêts de recherche sont variés. De la traduction scientifique et technique à la traduction littéraire, en passant par la terminologie, la traduction automatique, l'interprétation, l'histoire de la traduction, la pédagogie de la traduction, la lexicographie bilingue, la réception des traductions à l'étranger, etc. (Mareschal, 2005, p. 259)

L'enseignement de la traduction est un thème qui, en sol canadien, a donné lieu au fil des ans à des mémoires de maîtrise, des thèses de doctorat et des numéros spéciaux de revues. Pourtant, aucun chercheur canadien ne semble consacrer ses travaux de recherche uniquement à ce thème. C'est dans ce sens que j'estime qu'il s'agit d'un domaine «orphelin ", au même titre que les maladies orphelines, ces maladies rares ${ }^{7}$ auxquelles très peu de médecins et de chercheurs s'intéressent exclusivement, et auxquelles les sociétés pharmaceutiques et les gouvernements, principales sources de financement de la recherche médicale, ne s'intéressent guère davantage compte tenu du

7. En médecine, les maladies rares touchent 1 patient sur 2000 (Orphanet, 2012, n.p.; Regroupement québécois des maladies orphelines, 2015, n.p.), et les taux d'incidence peuvent varier d'une région à autre. 
marché très réduit pour les premières et du poids démographique peu significatif pour les seconds. Ainsi, même si l'enseignement de la traduction n'est pas un thème «rare», il est néanmoins «orphelin»: à ma connaissance, il n'existe pas au Canada de groupe de recherche sur l'enseignement de la traduction pas plus qu'il n'existe de projets de recherche menés par des professeurs-chercheurs et soutenus financièrement par les organismes subventionnaires fédéraux ou provinciaux. Jean Delisle a fait le même constat en 2007 :

Au Canada, on a fait des recherches originales en traduction dans bien de domaines : en traduction littéraire, en histoire, en terminologie, en traduction théâtrale et sur la conception féministe de la traduction. Assez peu de chercheurs, toutefois, ont fait de l'enseignement de la traduction leur principal domaine d'intérêt. Dans l'édition 20062007 du Répertoire des membres de l'Association canadienne de traductologie, une dizaine de chercheurs seulement sur 161 mentionnent explicitement ce champ de recherche [...]. (p. 83)

L'intérêt pour les questions relatives à l'enseignement de la traduction au Canada est pourtant manifeste. On peut le mesurer, entre autres, par le nombre de thèses de doctorat (cf., entre autres, Valentine, 1996; Williams, 2001; Fiola, 2003; Echeverri, 2008; Gardy, 2015; Kumbe, 2017) et de mémoires de maîtrise (cf., entre autres, Mascarenhas, 2011; Calderón Contreras, 2012; Marchand, 2012; St-Pierre, 2014; Saint-André, 2015; Arana, 2017) qui, depuis une vingtaine d'années, ont pris ces questions comme toile de fond. Par ailleurs, les revues de traductologie canadiennes Meta et $T T R$ ont publié au cours des trente dernières années six numéros portant spécifiquement sur l'enseignement de la traduction, soit trois numéros chacune :

- L'enseignement de la traduction au Canada (Meta, vol. 33, n 2; 1988), sous la direction de Robert Larose;

- La pédagogie de la traduction: questions actuelles (TTR, vol. V, $\mathrm{n}^{\circ} 1$; 1992), sous la direction de Jane Koustas;

- Traduction et enseignement (Meta, vol. 48, n 3; 2003), sous la direction d'Egan Valentine, Zélie Guével et Georges L. Bastin;

- Enseignement de la traduction dans le monde (Meta, vol. 50, n 1; 2005), sous la direction de Christian Balliu;

- La formation en traduction: pédagogie, docimologie et technologie (TTR, vol. XXI, $\left.\mathrm{n}^{\circ} 1 ; 2008\right)$, sous la direction de Georges L. Bastin et Marco A. Fiola; 
- La formation en traduction: pédagogie, docimologie et technologie (TTR, vol.XXI, nº 2; 2008), sous la direction de Marco A. Fiola et Georges L. Bastin.

À l'exception du numéro dirigé par Christian Balliu, tous les autres ont été dirigés par des chercheurs canadiens. De plus, parmi les collaborateurs de ces numéros, on compte de nombreux chercheurs qui enseignent ou qui ont enseigné dans des programmes de formation à la traduction d'universités canadiennes: MarieChristine Aubin, Lynne Bowker, Annie Brisset, Jeanne Dancette, Jean Delisle, Ginette Demers, Marco A. Fiola, Barbara Folkart, Jean-Claude Gémar, Jean-Marc Gouanvic, Paul A. Horguelin, Christine Klein-Lataud, Geneviève Mareschal, Geneviève Quillard, Roda P. Roberts, Sylvie Vandaele, Judith Woodsworth, etc.

Comme on peut le constater, les sujets relatifs à la formation des traducteurs suscitent l'intérêt de chercheurs établis et de jeunes chercheurs en contexte canadien. Cependant, ces chercheurs ne font pas pour autant de l'enseignement de la traduction leur principal champ de recherche, et l'on peut certes se demander pourquoi, surtout si l'on garde à l'esprit les propos de Daniel Gouadec cités en début d'article : «[a]nyone who writes a thesis on such subjects [training translators and training technical translators] may not expect to get excessively fast promotion ». Une étude serait nécessaire, par exemple, pour connaître les raisons qui mènent les doctorants qui s'intéressent à l'enseignement de la traduction à changer de sujet de recherche une fois qu'ils deviennent professeurs ou professeurs-chercheurs. En attendant qu'une telle étude soit réalisée, je suis d'avis que les futurs traductologues doivent être sensibilisés à la pertinence de la recherche sur l'enseignement de la traduction. Cette démarche est déjà entamée avec la création du séminaire «Formation des formateurs en traduction» offert aux étudiants inscrits à la maîtrise et au doctorat en traduction à l'Université de Montréal. Ce séminaire remplit une double fonction. Premièrement, il permet aux futurs traductologues d'acquérir des connaissances de base sur la formation des traducteurs et de prendre conscience qu'on ne s'improvise pas enseignant (de la même façon qu'on ne s'improvise pas traducteur). Deuxièmement, il expose les étudiants à une riche bibliographie qui leur donne un aperçu de la tradition canadienne en enseignement de la traduction et leur permet de s'approprier des connaissances produites sur le sujet tant au Canada qu'à l'étranger. Parmi les étudiants actuels se trouvent les prochaines générations de chercheurs en traductologie 
et de formateurs des traducteurs. Ce sont eux qui auront bientôt la responsabilité de concevoir, d'organiser et de gérer les programmes de formation, tout en assurant la production de connaissances en enseignement de la traduction et la qualité de la formation des traducteurs. C'est en effet par la production de connaissances relatives à l'enseignement de la traduction que les professeurs-chercheurs justifient leur rôle de formateurs, initialement assumé par les associations professionnelles. De plus, la possibilité de produire des connaissances sur la formation des traducteurs explique le contrôle exercé par les universités sur cette formation. Comme Sherry Simon le déclarait en 1989, l'étude de la traduction au Québec doit répondre à la fois aux besoins criants en formation de traducteurs ainsi qu'aux désirs de consolider et de promouvoir la traduction et les traducteurs professionnels (p. 11).

\section{Conclusion}

La formation des traducteurs au Canada a établi ses assises sur deux grands piliers méthodologiques: la dissociation des langues et le repérage des difficultés de traduction. Elle s'est fondée sur l'idée d'induire "des faits concrets de langage les règles ou les lois générales régissant la pratique de la traduction, et deuxième trait typique de cette tradition, d'axer cette réflexion sur les besoins de la formation en aval et en amont de carrière de traducteur» (Delisle, 1990, p. 65). Elle a donné lieu à une tradition qui, suivant la hiérarchie proposée par Lefevere (1977), trouve ses précurseurs parmi des professionnels devenus formateurs. Ces précurseurs ont montré le chemin aux pionniers responsables de la conception de son manifeste et d'un appareil théorique sur lequel ils ont bâti leur réputation internationale. Les pionniers ont cédé la place au maître, qui a continué sur leur lancée en créant un pont entre la traduction et les sciences de l'éducation. Le succès du maître est tel que la tradition canadienne de l'enseignement de la traduction cherche encore des disciples prêts à adopter de manière systématique et soutenue des efforts de recherche capables de mettre la formation des traducteurs au diapason des progrès faits par les sciences de l'éducation et par la profession.

La connaissance de cette tradition est importante étant donné qu'elle a pu se développer pour répondre aux besoins de la société canadienne en général, et québécoise en particulier. La production de connaissances scientifiques assure à l'université une partie du contrôle 
qu'elle exerce sur la formation des traducteurs. Pour maintenir ce contrôle, la communauté traductologique et universitaire en général devrait favoriser la création d'un environnement dans lequel des chercheurs pourraient se concentrer exclusivement à la recherche en enseignement de la traduction, dans le contexte de programmes de recherche qui se maintiennent dans le temps. La traduction est entrée à l'université derrière les cohortes d'étudiants à la recherche d'une formation professionnelle. À l'université, la traduction est devenue un champ de recherche autonome, une discipline, et elle s'y est épanouie.

Grâce aux efforts des universitaires qui ont provoqué l'émergence de la tradition canadienne en enseignement de la traduction, les formateurs d'aujourd'hui disposent d'une boîte à outils bien garnie pour accomplir leur tâche. La traduction raisonnée comme méthode d'initiation à la traduction des textes pragmatiques offre un exemple éloquent de l'importante contribution que les traductologues canadiens ont apporté à l'enseignement de la traduction, toutes traditions confondues. Conçu sur la base d'une tradition initiée par Georges Panneton en 1945, ce manuel a fait date grâce à sa pertinence et à son adéquation au rôle sociolinguistique que la traduction doit jouer au Canada. La traduction raisonnée célébrera bientôt son quarantième anniversaire. Elle a pris des rides, tout naturellement, et il sera bientôt temps de réviser et de mettre à jour le fondement pédagogique qui a contribué à son succès, l'approche d'enseignement par objectifs d'apprentissage.

\section{Références}

Arana, Cristina (2017). Description des stratégies d'enseignement dans la formation des traducteurs: le point de vue des étudiants. Mémoire de maîtrise, Département de linguistique et de traduction, Université de Montréal. Inédit.

Bloom, Benjamin, dir. (1975). Taxonomie des objectifs pédagogiques. Tome 1 : domaine cognitif. Trad. Marcel Lavallée. Québec, Les Presses de l'Université du Québec.

Calderón Contreras, Elisa (2012). Que faut-il conclure des évaluations réalisées par les encadreurs du Bureau de la traduction? La formation universitaire des traducteurs bacheliers récemment diplômés et son appréciation par l'industrie langagière canadienne. Thèse de maîtrise, École de traduction et d'interprétation, Université d'Ottawa. Inédit.

Daviault, Pierre (1931). L'expression juste en traduction. Notes de traduction, première série. Montréal, Éditions Albert Lévesque. 
Daviault, Pierre (1963). Langage et traduction. Ottawa, Bureau fédéral de traduction.

Delisle, Jean (1980). L'analyse du discours comme méthode de traduction. Initiation à la traduction française de textes pragmatiques anglais. Théorie et pratique. Ottawa, Les Presses de l'Université d'Ottawa.

Delisle, Jean (1990). Les Alchimistes des langues. La Société des traducteurs du Québec (1940-1990). Ottawa, Les Presses de l'Université d'Ottawa.

Delisle, Jean (1992). «Les manuels de traduction: essai de classification». TTR, 5, 1, p. 17-47.

Delisle, Jean (1993). La traduction raisonnée. Manuel d'initiation à la traduction professionnelle anglais-français. Ottawa, Les Presses de l'Université d'Ottawa.

Delisle, Jean (2003). La traduction raisonnée. Manuel d'initiation à la traduction professionnelle anglais-français. $2^{\mathrm{e}}$ éd. Ottawa, Les Presses de l'Université d'Ottawa.

Delisle, Jean (2007). «La formation de traducteurs et de terminologues en contexte canadien». Intertext, 3/4, p. 82-94.

Delisle, Jean et Marco A. Fiola (2013). La traduction raisonnée. Manuel d'initiation à la traduction professionnelle anglais-français. $3^{\mathrm{e}}$ éd. Ottawa, Les Presses de l'Université d'Ottawa.

Doughty, Howard (2006). «Blooming Idiots: Educational Objectives, Learning Taxonomies and the Pedagogy of Benjamin Bloom». College Quarterly, 9, 4, n.p. Disponible à <https://eric.ed.gov/?id=EJ835427> [consulté le 20 août 2018].

Dubuc, Robert (1994). En français dans le texte. Montréal, Linguatech.

Dubuc, Robert (2000). En français dans le texte. $2^{\mathrm{e}}$ éd. Montréal, Linguatech.

Durieux, Christine (2005). «L'enseignement de la traduction : enjeux et démarches». Meta, 50, 1, p. 36-47.

Echeverri, Álvaro (2008). Métacognition, apprentissage actif et traduction: l'apprenant de traduction, agent de sa propre formation. Thèse de doctorat, Département de linguistique et de traduction, Université de Montréal. Inédit.

Fiola, Marco A. (2003). La notion de programme en didactique de la traduction professionnelle: le cas du Canada. Thèse de doctorat, École Supérieure d'Interprètes et de Traducteurs, Université Paris III-Sorbonne Nouvelle. Inédit.

Gardy, Philippe (2015). L'évaluation en didactique de la traduction et l'intégration des outils technopédagogiques: étude qualitative et expérimentation. Thèse de doctorat, Département de langues, linguistique et traduction, Université Laval. Inédit.

Gauthier, Clermont et Maurice Tardif, dir. (2012). La pédagogie. Théories et pratiques de l'Antiquité à nos jours. $3^{\mathrm{e}}$ éd. Montréal, Gaëtan Morin Chenelière Éducation. 
Gimeno Sacristán, José (1982). La pedagogía por objetivos. Obsesión por la eficiencia. Madrid, Morata.

Gingras, Jules-Fabien (1860). Recueil des expressions vicieuses et des anglicismes les plus fréquents. Québec, E.R. Fréchette.

Gouadec, Daniel (2003). «Notes on Translation Training (Replies to a Questionnaire)». In A. Pym et al., dir. Innovation and E-Learning in Translator Training. Tarragone, Intercultural Studies Group, Universitat Rovira i Virgilii, p. 11-19. Disponible à <http://www.intercultural.urv. cat/media/upload/domain_317/arxius/Innovation/innovation_book. pdf> [consulté le 30 juillet 2018].

Horguelin, Paul (1984). «La traduction à l'ère des communications». In Société des traducteurs du Québec et Conseil de la langue française, Actes du colloque Traduction et qualité de langue. Québec, Conseil de la langue française, p. 24-35.

Hurtado Albir, Amparo, dir. (1999). Enseñar a traducir. Metodología en la formación de traductores e intérpretes. Madrid, Edelsa.

Hurtado Albir, Amparo (2015). Aprender a traducir del francés al español: Competencias y tareas para la iniciación a la traducción. Madrid, Edelsa.

Jones, Beau F. et Lorna Idol, dir. (1990). Dimensions of Thinking and Cognitive Instruction. Hillsdale [N. J.], Lawrence Erlbaum Associates.

Juhel, Denis (1982). Bilinguisme et traduction au Canada : rôle sociolinguistique du traducteur. Québec, Centre international de recherche sur le bilinguisme.

Kelly, Dorothy (2005). A Handbook for Translator Trainers. Manchester, St. Jerome.

Kiraly, Donald (1995). Pathways to Translation. Kent, Kent State University Press.

Kumbe, Kornebari (2017). La formation des traducteurs en Amérique du Nord et en Europe occidentale au XXI siècle. Thèse de doctorat, Département de linguistique et de traduction, Université de Montréal. Inédit.

Lavallée, François (2005). Le traducteur averti: pour des traductions idiomatiques. Montréal, Linguatech.

Lavallée, François (2016). Le traducteur encore plus averti : pour des ornières de traduction. Montréal, Linguatech.

Lefevere, André (1977). Translating Literature: The German Tradition from Luther to Rosenzweig. Assen/Amsterdam, Van Gorcun.

Louis, Roland, France Jutras et Hélène Hensler (1996). «Des objectifs aux compétences : implications pour l'évaluation de la formation initiale des maîtres». Revue canadienne de l'éducation, 21, 4, p. 414-432.

Mager, Robert F. (1977). Comment définir des objectifs pédagogiques. Trad. G. Decote. Paris, Bordas.

Maguire, Thomas (1841). Manuel des difficultés les plus communes de la langue 
française, adapté au jeune âge, et suivi d'un Recueil de locutions vicieuses, Québec, Fréchette et Cie.

Marchand, Chantale (2012). De la pédagogie dans les manuels de traduction: Analyse comparative des manuels anglais-français publiés en Amérique du Nord et en Europe depuis 1992. Mémoire de maîtrise, Département de linguistique et de traduction, Université de Montréal. Inédit.

Mareschal, Geneviève (2005). «L'enseignement de la traduction au Canada». Meta, 50, 1, p. 250-262.

Mascarenhas, Alice (2011). Stratégies d'enseignement du curriculum opératoire que les formateurs affirment appliquer en vue de cibler les lacunes en langue seconde dans les baccalauréats en traduction au Québec. Mémoire de maîtrise. Département des sciences de l'éducation, Université du Québec en Outaouais. Inédit.

Newmark, Peter (1991). About Translation. Clevedon, Multilingual Matters.

Orphanet (2012). «À propos des maladies rares». Orphanet. Disponible à $<$ https://www.orpha.net/consor/cgi-bin/Education_AboutRareDiseases. php?lng=FR> [consulté le 30 juillet 2018].

Panneton, Georges (1945). La transposition : principe de la traduction. Son rôle essentiel dans l'interprétation de la pensée, sa valeur de base technique. Thèse de maîtrise, Faculté des lettres, Université de Montréal. Inédit.

Pym, Anthony et al., dir. (2003). Innovation and E-Learning in Translator Training. Tarragone, Intercultural Studies Group, Universitat Rovira i Virgilii. Disponible à <http://www.intercultural.urv.cat/media/upload/ domain_317/arxius/Innovation/innovation_book.pdf> [consulté le 30 juillet 2018].

Regroupement québécois des maladies orphelines (2015). «Information sur les maladies rares et orphelines». Disponible à <https://rqmo. org/information-sur-les-maladies-rares-et-orphelines/> [consulté le 28 août 2018].

Saint-André, Louise (2015). Quelle formation donner aux traducteurspostéditeurs de demain? Thèse de maîtrise, École de traduction et d'interprétation, Université d'Ottawa. Inédit.

Simon, Sherry (1989). L'inscription sociale de la traduction au Québec. Montréal, Office de la langue française.

St-Pierre, Carine (2014). Le modèle de compétences comme tronc commun des programmes de traduction. Thèse de maîtrise, École de traduction et d'interprétation, Université d'Ottawa. Inédit.

Tardif, Jacques (1997). Pour un enseignement stratégique: l'apport de la psychologie cognitive. Montréal, Les Éditions Logiques.

Valentine, Egan (1996). Traductologie, traduction et formation: vers une modélisation de la formation en traduction - l'expérience canadienne. Thèse de doctorat, Département de linguistique et de traduction, Université de Montréal. Inédit. 
Van der Maren, Jean-Marie (2003). La recherche appliquée en pédagogie: des modèles pour l'enseignement. Bruxelles, De Boeck.

Vinay, Jean-Paul et Jean Darbelnet (1958). Stylistique comparée du français et de l'anglais : méthode de traduction. Montréal, Beauchemin.

Williams, Malcolm (2001). An Argumentation-Centred Approach to Translation Quality Assessment. Thèse de doctorat, École de traduction et d'interprétation, Université d'Ottawa.

Williams,Malcolm (2004). Translation Quality Assessment:An ArgumentationCentred Approach. Ottawa, Les Presses de l'Université d'Ottawa.

Álvaro Echeverri Département de linguistique et de traduction Université de Montréal Montréal (Québec) Canada a.echeverri@umontreal.ca 